\title{
Modelling the Transmission Dynamics of COVID-19 in Six High- Burden Countries
}

\author{
Azizur Rahman $\mathbb{D i D}^{1}$ and Md Abdul Kuddus $\mathbb{D i D}^{2,3}$ \\ ${ }^{1}$ Data Science Research Unit, School of Computing and Mathematics, Charles Sturt University, Wagga Wagga, NSW 2678, Australia \\ ${ }^{2}$ Australian Institute of Tropical Health and Medicine, James Cook University, Townsville, QLD 4810, Australia \\ ${ }^{3}$ Department of Mathematics, University of Rajshahi, Rajshahi 6205, Bangladesh
}

Correspondence should be addressed to Azizur Rahman; azrahman@csu.edu.au

Received 15 April 2020; Revised 11 December 2020; Accepted 17 May 2021; Published 2 June 2021

Academic Editor: Fernando José Dias

Copyright ( 2021 Azizur Rahman and Md Abdul Kuddus. This is an open access article distributed under the Creative Commons Attribution License, which permits unrestricted use, distribution, and reproduction in any medium, provided the original work is properly cited.

\begin{abstract}
The new Coronavirus Disease 19, officially known as COVID-19, originated in China in 2019 and has since spread worldwide. We presented an age-structured Susceptible-Latent-Mild-Critical-Removed (SLMCR) compartmental model of COVID-19 disease transmission with nonlinear incidence during the pandemic period. We provided the model calibration to estimate parameters with day-wise COVID-19 data, i.e., reported cases by worldometer from $15^{\text {th }}$ February to $30^{\text {th }}$ March 2020 in six high-burden countries, including Australia, Italy, Spain, the USA, the UK, and Canada. We estimate transmission rates for each country and found that the country with the highest transmission rate is Spain, which may increase the new cases and deaths than the other countries. We found that saturation infection negatively impacted the dynamics of COVID-19 cases in all the six high-burden countries. The study used a sensitivity analysis to identify the most critical parameters through the partial rank correlation coefficient method. We found that the transmission rate of COVID-19 had the most significant influence on prevalence. The prediction of new cases in COVID-19 until 30 th April 2020 using the developed model was also provided with recommendations to control strategies of COVID-19. We also found that adults are more susceptible to infection than both children and older people in all six countries. However, in Italy, Spain, the UK, and Canada, older people show more susceptibility to infection than children, opposite to the case in Australia and the USA. The information generated from this study would be helpful to the decision-makers of various organisations across the world, including the Ministry of Health in Australia, Italy, Spain, the USA, the UK, and Canada, to control COVID-19.
\end{abstract}

\section{Introduction}

Following the outbreak of the novel Severe Acute Respiratory Syndrome Coronavirus-2 (SARS-CoV-2), COVID-19 constitutes a persistent and significant public health problem worldwide. As of $30^{\text {th }}$ March 2020, the ongoing global pandemic outbreak of COVID-19 has spread to at least 180 countries and territories, including Australia, Italy, Spain, the USA, the UK, and Canada, and resulted in approximately 946,876 cases of COVID-19 and 48,137 deaths [1]. In Australia, Italy, Spain, the USA, the UK, and Canada, COVID-19 infections and deaths reached 4460, 101739, 87956, 163788, 22141, and 7448, as well as $30,11591,7716,3143,1408$, and 89 , with mortality ratios of nearly $0.67 \%, 11.39 \%$, $8.77 \%, 1.9 \%, 6.4 \%$, and $1.2 \%$, respectively [1]. Figure 1 shows the cumulative number of confirmed cases and deaths of COVID-19 in six selected countries from $15^{\text {th }}$ February to $30^{\text {th }}$ March 2020.

The highest burden of COVID-19 is reliant on the health system and depends on a quick and timely response to the pandemic. For example, in Italy, the first confirmed COVID-19 cases were on February 15, and then, after a few days, thousands of people were infected by COVID-19. The problem is not that the Italian government did not respond to the COVID-19. The problem is that it always responded slightly too slow and with slightly too much moderation. 


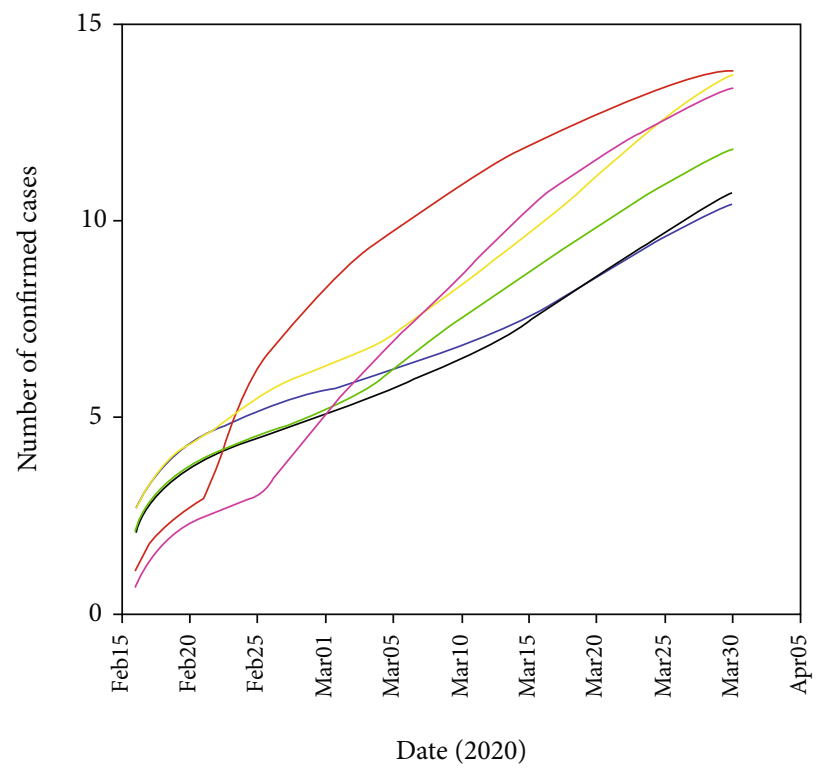

(a)

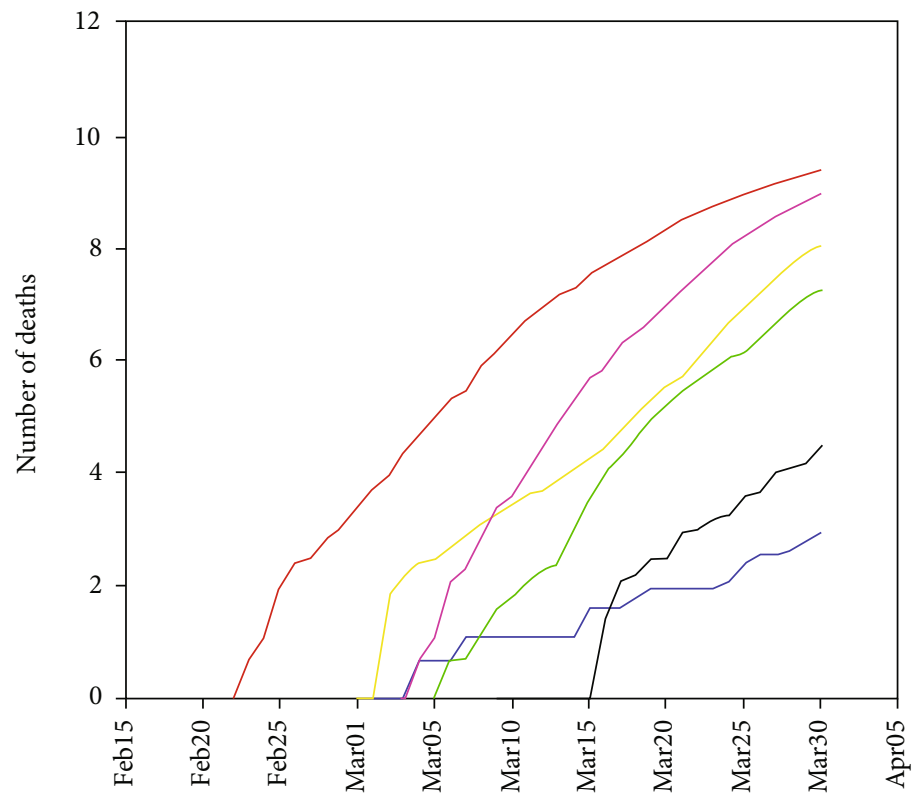

Date (2020)

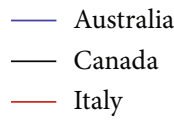

— Canada

- Italy

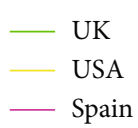

(b)

FIgURE 1: Graphs of six selected countries using a log scale: (a) cumulative number of COVID-19 cases and (b) cumulative number of COVID-19 deaths.

What has resulted in China reveals that quarantine, social distancing, and isolation of infected populations can contain the pandemic. This impact of the COVID-19 response in China is being advocated in many countries where COVID-19 is starting to spread. However, it is unclear whether other countries can implement the stringent measures China eventually adopted. Singapore and Hong Kong, both of which had severe acute respiratory syndrome (SARS) epidemics in 2002-2003, present concern and many lessons to other countries. In both places, COVID-19 has been maintained well to date, notwithstanding early cases, by early government progress and through social distancing patterns used by individuals.

A series of critical factors can lead to the outbreak of the COVID-19 pandemic. However, some of those factors seem 
to be poorly understood. Mathematical modelling is a powerful tool for infectious disease control that helps to accurately predict behaviour and understand infectious disease dynamics [2-4]. Many researchers have implemented mathematical modelling frameworks to gain insights into different infectious diseases [5-9]. Although models can range from very simple to highly complex, one of the most typical practices to improve understanding of infectious disease dynamics is the compartmental mathematical model [10].

In mathematical models, the incidence rate plays a vital role in the transmission of infectious diseases. The number of individuals who become infected per unit time is called the incidence rate in the epidemiology perspective [11]. Here, we consider the nonlinear incidence rate because the number of effective contacts between infective and susceptible individuals may saturate at high levels through the crowding of infective individuals [12]. This model is also used to calibrate and predict the number of COVID-19 case data in six countries, including Australia, Italy, Spain, the USA, the UK, and Canada, to estimate the model parameters. We assessed the impact of age structure on the dynamics of COVID-19 cases in all six burden countries. The study performed a sensitivity analysis to identify the essential model parameters that could support policymakers in controlling the COVID-19 outbreak in the selected countries. The model findings can be also helpful to many other countries which are dealing with the critical outbreak of COVID-19.

The rest of the paper is structured as follows: Section 2 presents model descriptions. Sections 3, 4, and 5, respectively, performed the model calibration and impact of saturation infection, the impact of age structure, and sensitivity analysis. Section 6 finalizes the paper with a brief discussion and concluding remarks.

\section{Model Description and Analysis}

We considered an SLMCR compartmental model with three age classes: children (0-14 years), adults (15-64 years), and older (over 64 years) of COVID-19 transmission with a nonlinear incidence between the following mutually exclusive compartments: $S(t)$ : susceptible individuals; $L(t)$ : latent individuals, representing those who are infected and have not yet developed active COVID-19; $M(t)$ : mild individuals who are both infected and infectious and have mild respiratory illness symptoms such as nasal congestion, runny nose, and a sore throat; $C(t)$ : critical individuals who are both infected and infectious and have severe symptoms including shortness of breath, chest discomfort, and bluish face; and $R(t)$ : recovered individuals who are previously infected but successfully recovered. Figure 2 depicts a typical SLMCR model.

Let the susceptible individuals be recruited at a constant rate $\Lambda$, and they may be infected at a time-dependent rate $\beta$ $(M+C) /(1+\alpha(M+C))$. Here, $\beta(M+C) /(1+\alpha(M+C))$ represents the saturated incidence rate, which tends to a saturated level when $(M+C)$ gets large. $\beta(M+C)$ measures the force of infection when the disease is entering a fully susceptible population, and $1 /(1+\alpha(M+C))$ measures the inhibition effect from the behaviour change of susceptible

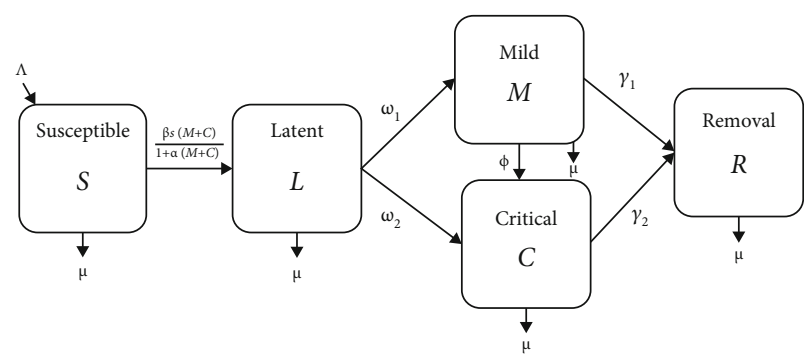

FIgURE 2: Flow chart of the SLMCR mathematical model showing the five states and the transitions in and out of each state. $S$ : susceptible population; $L$ : latent population (not yet symptomatic); $M$ : mild population (moderate symptom); $C$ : critical population (severe symptom); $R$ : removal population; $\Lambda$ : recruited rate; $\mu$ : death rate; $\beta$ : transmission rate; $\alpha$ : force of saturation infection; $\omega_{1}$ : progression rate from latent to a mild compartment; $\omega_{2}$ : progression rate from the latent critical compartment; $\gamma_{1}$ : recovery rate from mild to removal compartment; $\gamma_{2}$ : recovery rate from critical to removal compartment; $\phi$ : progression rate from mild to critical compartment due to the comorbidities with other diseases.

individuals when their number increases or from the effect of risk factors including a crowded environment of the infective individuals with $\alpha$ which determines the level at which the force of infection saturates. Individuals in different compartments suffer from natural death at the same constant rate $\mu$. All infected individuals move to the latently infected compartment, $L(t)$. Those with latent infection progress to mild and critical infections (the $M$ and $C$ compartments) due to reactivation of the latent infection at rate $\omega_{1}$ and $\omega_{2}$, respectively. However, some mild populations also move to the critical compartment due to the comorbidities with other diseases, including hypertension, diabetes, cardiovascular disease, and respiratory system disease [13]. A proportion of the mild and critical individuals recover through treatment and natural recovery rates $\gamma_{1}$ and $\gamma_{2}$, respectively, and move into the recovered compartment $R(t)$. In this case, we can express the model by the following five differential equations with an age-structured contact matrix, $\mathrm{P}$ :

$$
\begin{gathered}
\frac{\mathrm{d} S_{i}}{\mathrm{~d} t}=\Lambda-\frac{\beta S_{i}}{1+\alpha\left(M_{i}+C_{i}\right)} \sum_{j} \mathrm{P}_{i j}\left(M_{j}+C_{j}\right)-\mu S_{i} \\
\frac{\mathrm{d} L_{i}}{\mathrm{~d} t}=\frac{\beta S_{i}}{1+\alpha\left(M_{i}+C_{i}\right)} \sum_{j} P_{i j}\left(M_{j}+C_{j}\right)-\left(\omega_{1}+\omega_{2}+\mu\right) L_{i} \\
\frac{\mathrm{d} M_{i}}{\mathrm{~d} t}=\omega_{1} L_{i}-\left(\phi+\gamma_{1}+\mu\right) M_{i} \\
\frac{\mathrm{d} C_{i}}{\mathrm{~d} t}=\omega_{2} L_{i}+\phi M_{i}-\left(\gamma_{2}+\mu\right) C_{i} \\
\frac{\mathrm{d} R_{i}}{\mathrm{~d} t}=\gamma_{1} M_{i}+\gamma_{2} C_{i}-\mu R_{i}
\end{gathered}
$$

where $i$ and $j$ are the indices of the age classes and $\mathrm{P}$ represents the contact matrix. The matrix $\mathrm{P}$ has satisfied 


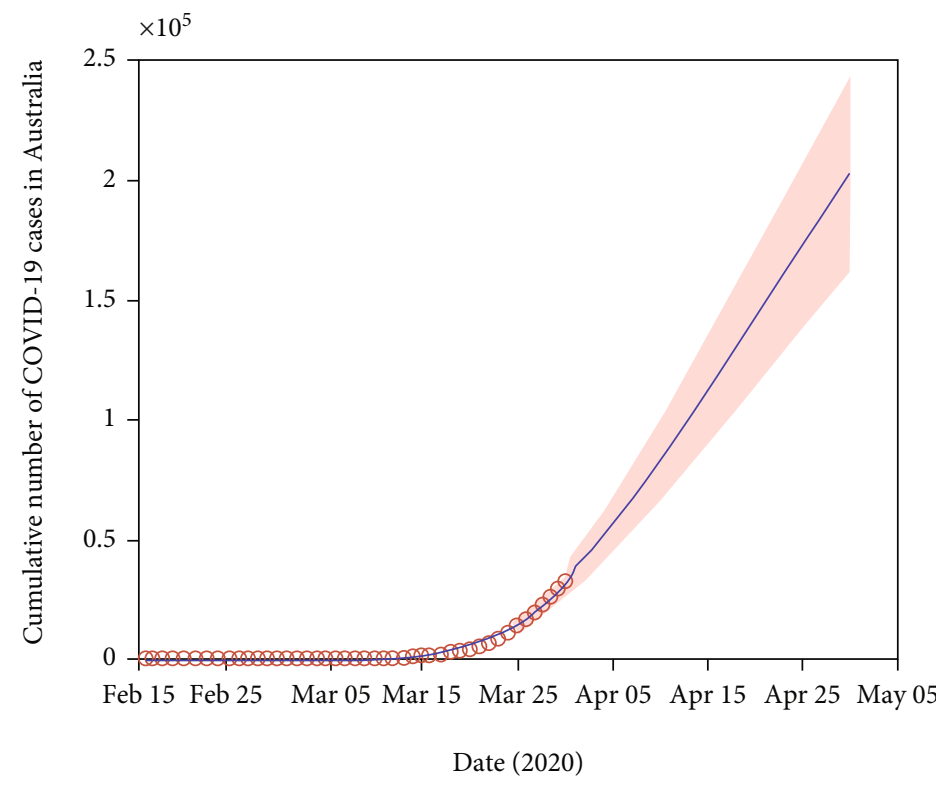

(a)

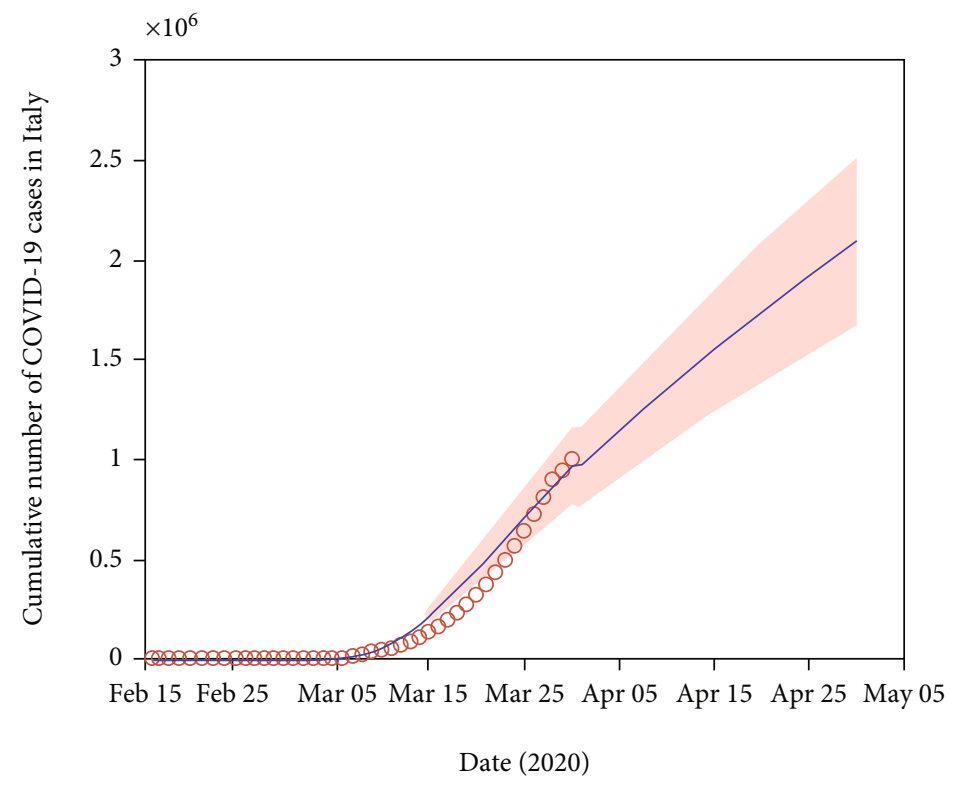

(b)

Figure 3: Continued. 


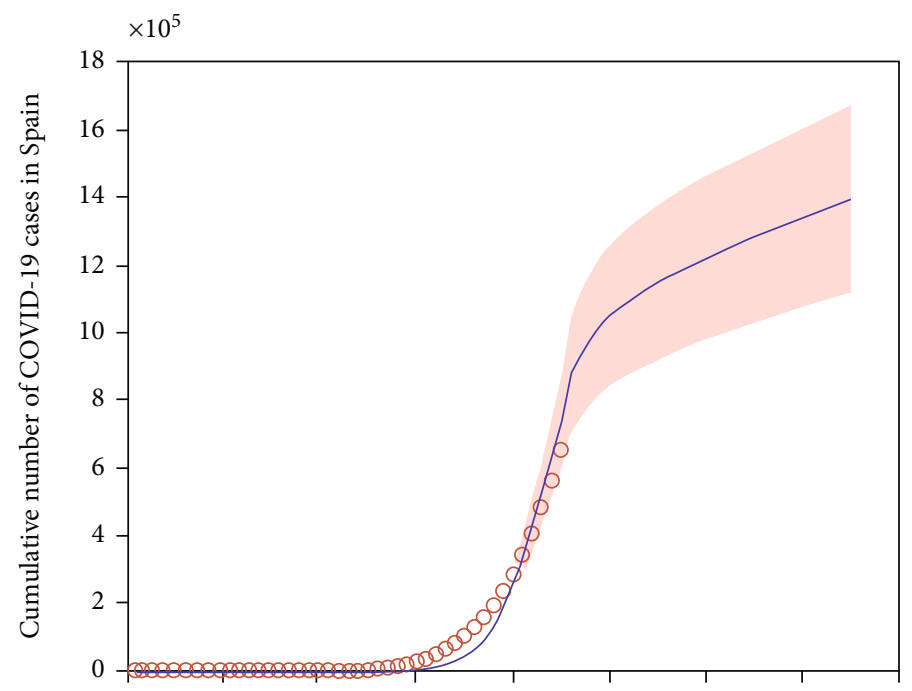

Feb 15 Feb 25 Mar 05 Mar 15 Mar 25 Apr 05 Apr 15 Apr 25 May 05

Date (2020)

(c)

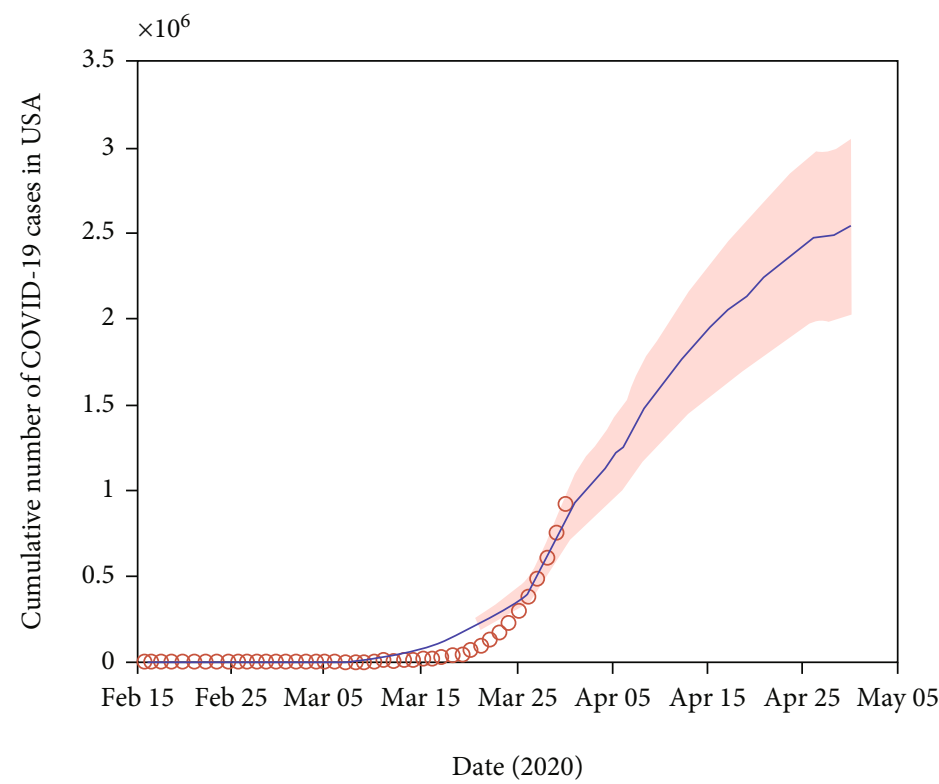

(d)

FIgURE 3: Continued. 


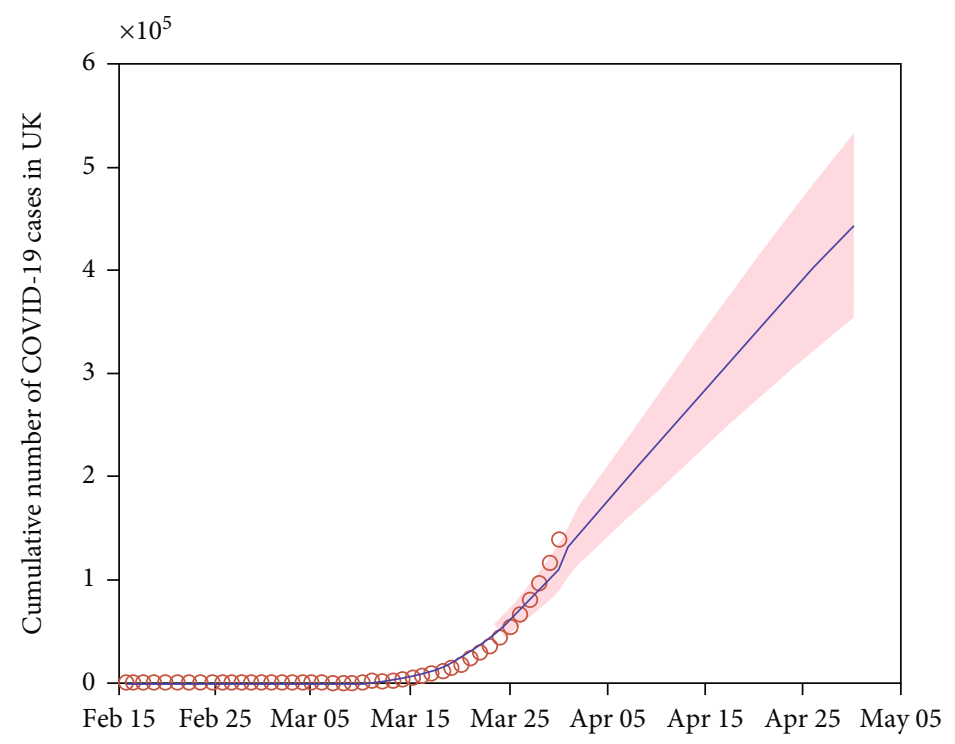

Date (2020)

(e)

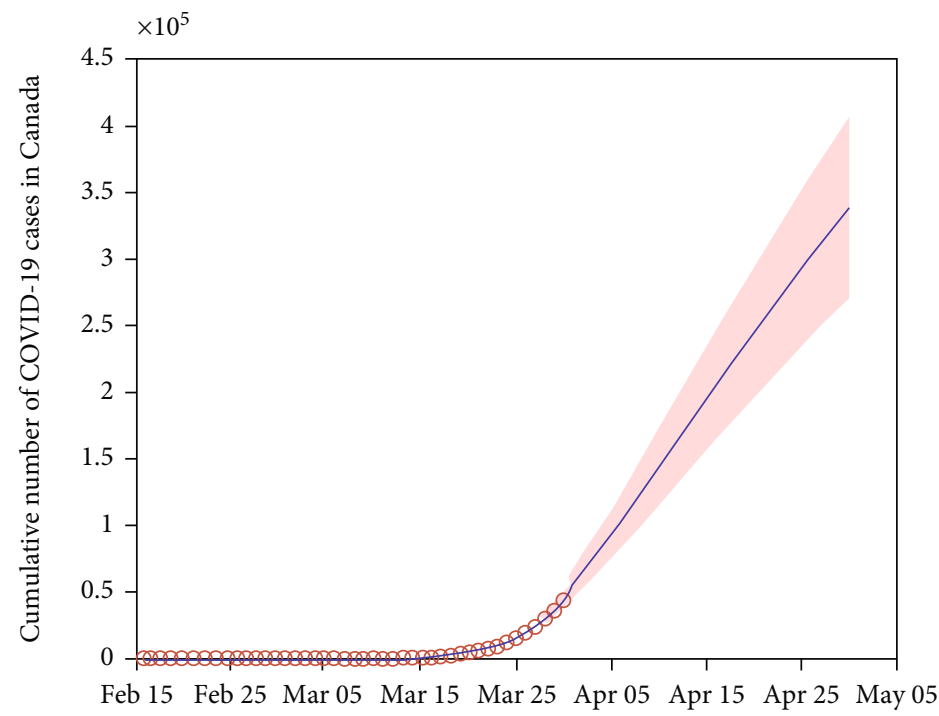

Date $(2020)$

(f)

FIGURE 3: Measured and predicted number of cumulative COVID-19 cases from $15^{\text {th }}$ February to $30^{\text {th }}$ March 2020 (red dot) in six different high-burden countries, (a) Australia, (b) Italy, (c) Spain, (d) the USA, (e) the UK, and (f) Canada, and the corresponding model (the bluesolid curve) with the $95 \%$ confidence interval (CI) measure in the rose-shaded limits.

reciprocity, meaning that within the population, the total time spent by the children with adults and older people, the adults with children and older people, and the older people with children and adults must be equal to the time spent by the adults and older people with children, the children and older people with adults, and the adults and children with older people.

Given the nonnegative initial conditions for the system above, it is straightforward to show that each of the state variables remains nonnegative for all $t>0$. Moreover, summing equations (1)-(5), we find that the size of the total population, $N_{i}(t)$, satisfies

$$
\frac{\mathrm{d} N_{i}(t)}{\mathrm{d} t} \leq \Lambda-\mu N_{i}
$$

Integrating the above inequality equation, we find

$$
N_{i}(t) \leq \frac{\Lambda}{\mu}+N_{i}(0) e^{-\mu t} .
$$


TABLE 1: Depiction and estimation of the model parameters for six countries.

\begin{tabular}{|c|c|c|c|c|}
\hline Countries & Parameters & Description & Estimated values & References \\
\hline \multirow{10}{*}{ Australia } & $N$ & Population in 2020 & $25,499,884$ & [15] \\
\hline & $\mu$ & Death rate & $\frac{1}{70} \mathrm{yr}^{-1}$ & {$[16]$} \\
\hline & $\beta$ & Transmission rate & $4.6214 \times 10^{-8}$ & Fitted \\
\hline & $\omega_{1}$ & Progression rate from $L$ to $M$ & 0.001 & Assumed \\
\hline & $\omega_{2}$ & Progression rate from $L$ to $C$ & 0.001 & Assumed \\
\hline & $\gamma_{1}$ & Recovery rate from $M$ to $R$ & 0.1 & Assumed \\
\hline & $\gamma_{2}$ & Recovery rate from $C$ to $R$ & 0.1 & Assumed \\
\hline & $\alpha$ & Infection saturation rate & 0.00001 & Assumed \\
\hline & $\phi$ & Comorbidity rate & 0.3 & Assumed \\
\hline & $\Lambda$ & Recruitment rate & 1 & \\
\hline \multirow{10}{*}{ Italy } & $N$ & Population in 2020 & $60,461,826$ & {$[17]$} \\
\hline & $\mu$ & Death rate & $\frac{1}{70} \mathrm{yr}^{-1}$ & {$[16]$} \\
\hline & $\beta$ & Transmission rate & $1.2292 \times 10^{-8}$ & Fitted \\
\hline & $\omega_{1}$ & Progression rate from $L$ to $M$ & 0.009 & Assumed \\
\hline & $\omega_{2}$ & Progression rate from $L$ to $C$ & 0.009 & Assumed \\
\hline & $\gamma_{1}$ & Recovery rate from $M$ to $R$ & 0.14 & Assumed \\
\hline & $\gamma_{2}$ & Recovery rate from $M$ to $R$ & 0.10 & Assumed \\
\hline & $\alpha$ & Infection saturation rate & 0.0000058 & Assumed \\
\hline & $\phi$ & Comorbidity rate & 0.3 & Assumed \\
\hline & $\Lambda$ & Recruitment rate & 1 & \\
\hline \multirow{10}{*}{ Spain } & $N$ & Population in 2020 & $46,754,778$ & {$[18]$} \\
\hline & $\mu$ & Death rate & $\frac{1}{70} \mathrm{yr}^{-1}$ & {$[16]$} \\
\hline & $\beta$ & Transmission rate & $3.3182 \times 10^{-7}$ & Fitted \\
\hline & $\omega_{1}$ & Progression rate from $L$ to $M$ & 0.00034 & Assumed \\
\hline & $\omega_{2}$ & Progression rate from $L$ to $C$ & 0.00034 & Assumed \\
\hline & $\gamma_{1}$ & Recovery rate from $M$ to $R$ & 0.3 & Assumed \\
\hline & $\gamma_{2}$ & Recovery rate from $M$ to $R$ & 0.3 & Assumed \\
\hline & $\alpha$ & Infection saturation rate & 0.0000004 & Assumed \\
\hline & $\phi$ & Comorbidity rate & 0.3 & Assumed \\
\hline & $\Lambda$ & Recruitment rate & 1 & \\
\hline \multirow{10}{*}{ USA } & $N$ & Population in 2020 & $331,002,651$ & {$[19]$} \\
\hline & $\mu$ & Death rate & $\frac{1}{70} \mathrm{yr}^{-1}$ & {$[16]$} \\
\hline & $\beta$ & Transmission rate & $3.1828 \times 10^{-7}$ & Fitted \\
\hline & $\omega_{1}$ & Progression rate from $L$ to $M$ & 0.02 & Assumed \\
\hline & $\omega_{2}$ & Progression rate from $L$ to $C$ & 0.01 & Assumed \\
\hline & $\gamma_{1}$ & Recovery rate from $M$ to $R$ & 0.02 & Assumed \\
\hline & $\gamma_{2}$ & Recovery rate from $C$ to $R$ & 0.008 & Assumed \\
\hline & $\alpha$ & Infection saturation rate & 0.00000266 & Assumed \\
\hline & $\phi$ & Comorbidity rate & 0.3 & Assumed \\
\hline & $\Lambda$ & Recruitment rate & 1 & \\
\hline
\end{tabular}


TABLe 1: Continued.

\begin{tabular}{|c|c|c|c|c|}
\hline Countries & Parameters & Description & Estimated values & References \\
\hline \multirow{10}{*}{ UK } & $N$ & Population in 2020 & $67,886,011$ & {$[20]$} \\
\hline & $\mu$ & Death rate & $\frac{1}{70} \mathrm{yr}^{-1}$ & {$[16]$} \\
\hline & $\beta$ & Transmission rate & $1.1526 \times 10^{-7}$ & Fitted \\
\hline & $\omega_{1}$ & Progression rate from $L$ to $M$ & 0.0003 & Assumed \\
\hline & $\omega_{2}$ & Progression rate from $L$ to $C$ & 0.0003 & Assumed \\
\hline & $\gamma_{1}$ & Recovery rate from $M$ to $R$ & 0.1 & Assumed \\
\hline & $\gamma_{2}$ & Recovery rate from $C$ to $R$ & 0.02 & Assumed \\
\hline & $\alpha$ & Infection saturation rate & 0.00001 & Assumed \\
\hline & $\phi$ & Comorbidity rate & 0.3 & Assumed \\
\hline & $\Lambda$ & Recruitment rate & 1 & \\
\hline \multirow{10}{*}{ Canada } & $N$ & Population in 2020 & $37,742,154$ & {$[21]$} \\
\hline & $\mu$ & Death rate & $\frac{1}{70} \mathrm{yr}^{-1}$ & {$[16]$} \\
\hline & $\beta$ & Transmission rate & $2.0731 \times 10^{-7}$ & Fitted \\
\hline & $\omega_{1}$ & Progression rate from $L$ to $M$ & 0.0003 & Assumed \\
\hline & $\omega_{2}$ & Progression rate from $L$ to $C$ & 0.0003 & Assumed \\
\hline & $\gamma_{1}$ & Recovery rate from $M$ to $R$ & 0.2 & Assumed \\
\hline & $\gamma_{2}$ & Recovery rate from $C$ to $R$ & 0.2 & Assumed \\
\hline & $\alpha$ & Infection saturation rate & 0.00001 & Assumed \\
\hline & $\phi$ & Comorbidity rate & 0.3 & Assumed \\
\hline & $\Lambda$ & Recruitment rate & 1 & \\
\hline
\end{tabular}

The result shows the total population size $N_{i}(t)$ bounded in this case, and naturally, it follows that each of the compartment states (i.e., $S, L, M, C$, and $R$ ) are also bounded.

\section{Estimation of Model Parameters}

This section estimated the model parameters based on the available six countries' COVID-19 reported case data from http://worldometers.info [1]. Figure 3 presents the curve of cumulative confirmed COVID-19 cases each day from the $15^{\text {th }}$ February to $30^{\text {th }}$ March 2020 in Australia, Italy, Spain, the USA, the UK, and Canada. In order to parameterize the model (1)-(5), we obtained some of the parameter values from the literature (see Table 1). Others were estimated or fitted from the data. We obtained the bestfitted parameter values by minimizing the error using the least-square method between the COVID-19 case data and the solution of the proposed model (1)-(5) (see the blue-solid graph in Figure 3). The model was fitted in MATLAB using the multistart algorithm with 1000 starting points [14]. By keeping the model-convergence results, we estimated the confidence intervals of the model with the normal distribution assumption.
The objective function used in the parameter estimation is as follows:

$$
\widehat{\theta}=\operatorname{argmin} \sum_{i=1}^{n}\left(\left(\omega_{1}+\omega_{2}\right) L-\operatorname{data}_{t_{i}}\right)^{2},
$$

where data $t_{t_{i}}$ denotes the COVID-19 data and $\left(\omega_{1}+\omega_{2}\right) L$ is the corresponding model solution at time $t_{i}$, while $n$ is the number of available actual data points. Findings reveal that the proposed model is well-fitted with the data.

The prediction results from the model are also depicted in Figure 3 to assist in the evidence-based decision-making process. For example, predicted results could assist politicians or decision-makers in the health department of Australia, Italy, Spain, the USA, the UK, and Canada for planning for their health systems' need. They can then implement measures regarding staff resources and hospital beds to meet the challenges of this difficult time. However, if the number of infected individuals follows this trend for the next month, there will be more than 200,000 in Australian, 200,000,000 in Italian, $140,000,000$ in Spanish, 250,000,000 in USA, $40,000,000$ in UK, and 300,000 in Canadian patients infected by $30^{\text {th }}$ April 2020 as shown in Figure 3. 


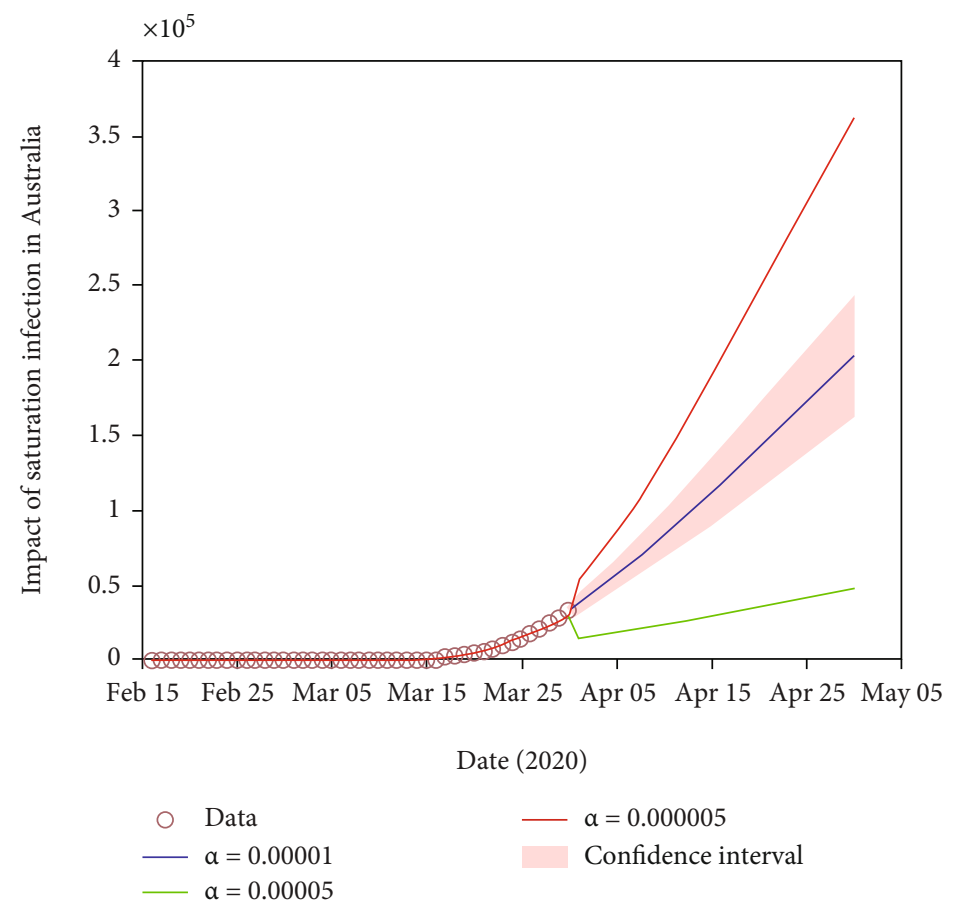

(a)

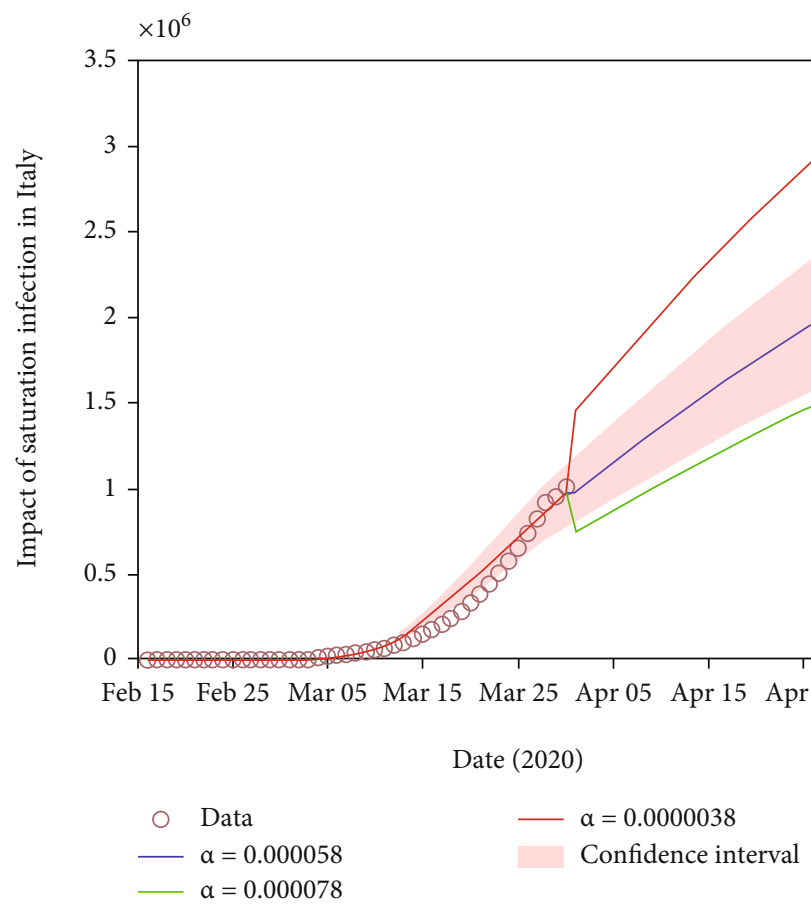

(b)

FIgURE 4: Continued. 


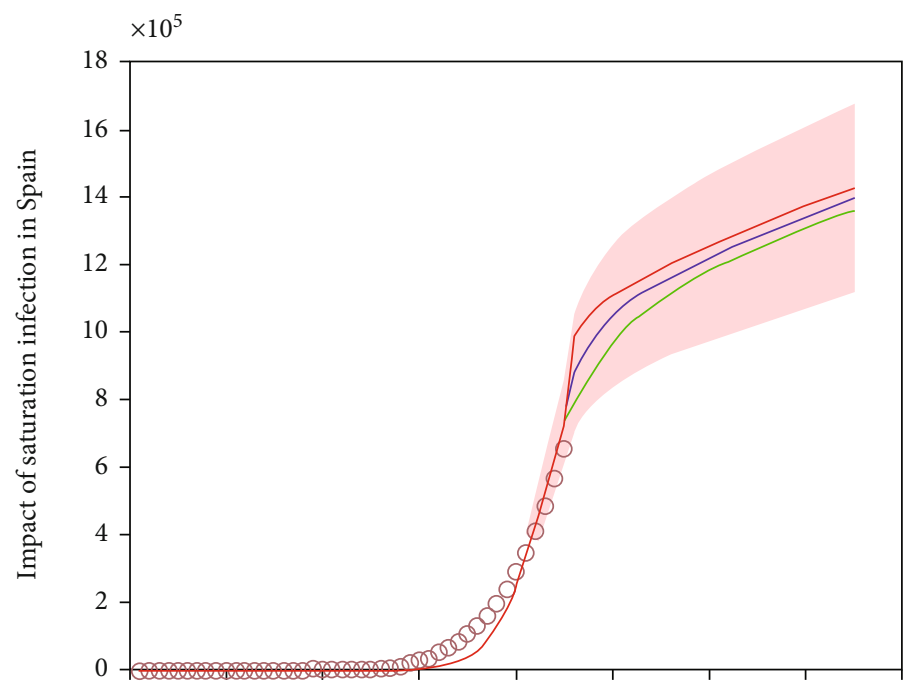

Feb 15 Feb 25 Mar 05 Mar 15 Mar 25 Apr 05 Apr 15 Apr 25 May 05

Date (2020)

Data
$-a=0.0000004$
$\square$
$\alpha=0.0000008$

$\times 10^{6}$

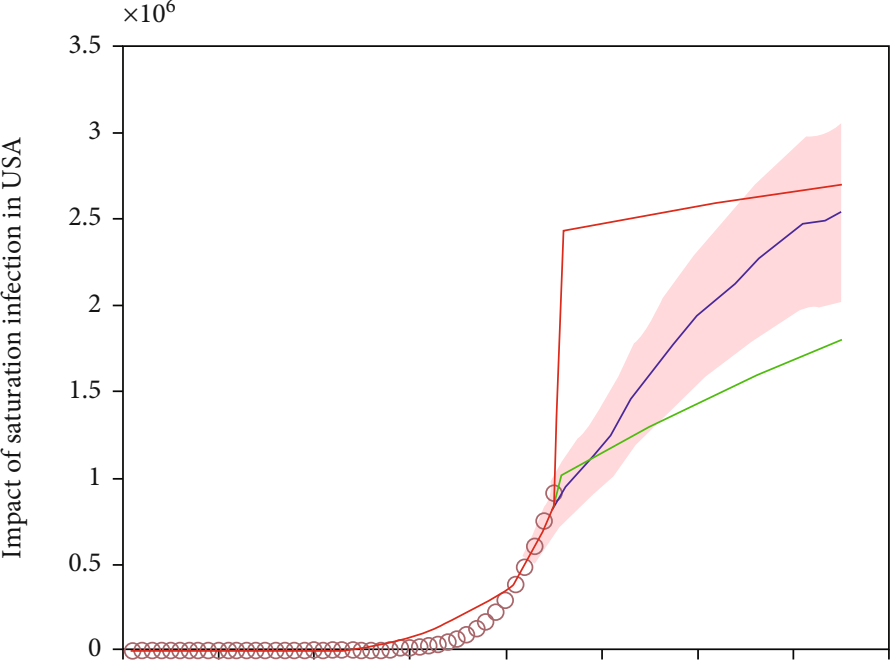

Feb 15 Feb 25 Mar 05 Mar 15 Mar 25 Apr 05 Apr 15 Apr 25 May 05

○ Data

$-\alpha=0.00000266$ $\alpha=0.00000566$
Date (2020)

$-\alpha=0.00000005$

Confidence interval

(c)

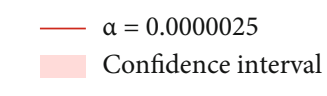

(d)

FIgURe 4: Continued. 


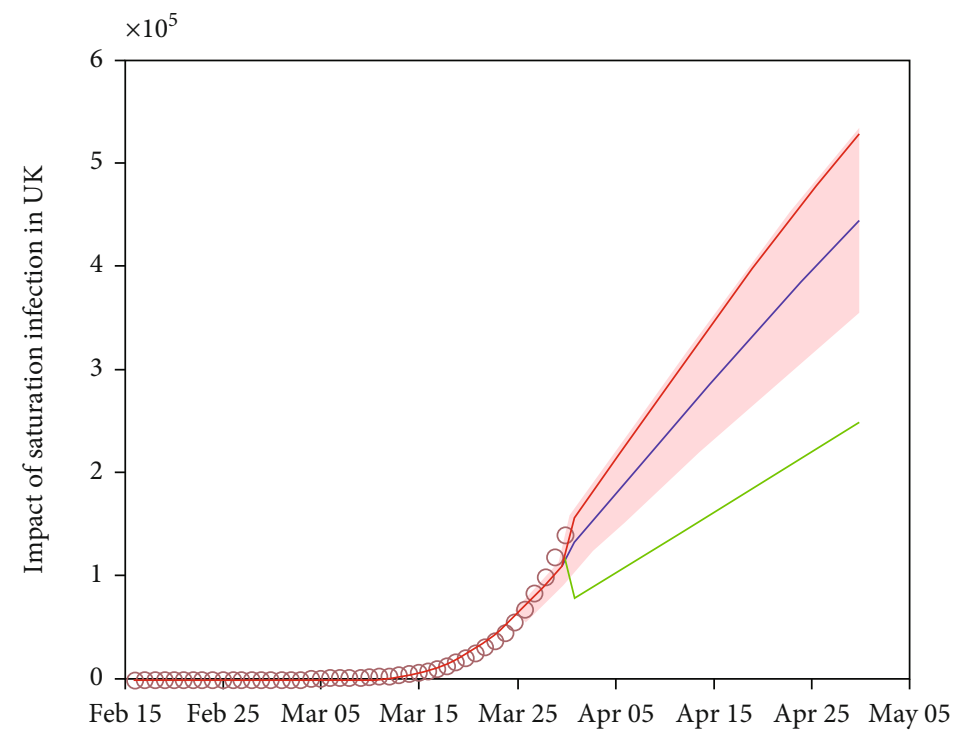

Date (2020)

0 Data

$-\alpha=0.00001$

$-\alpha=0.00002$

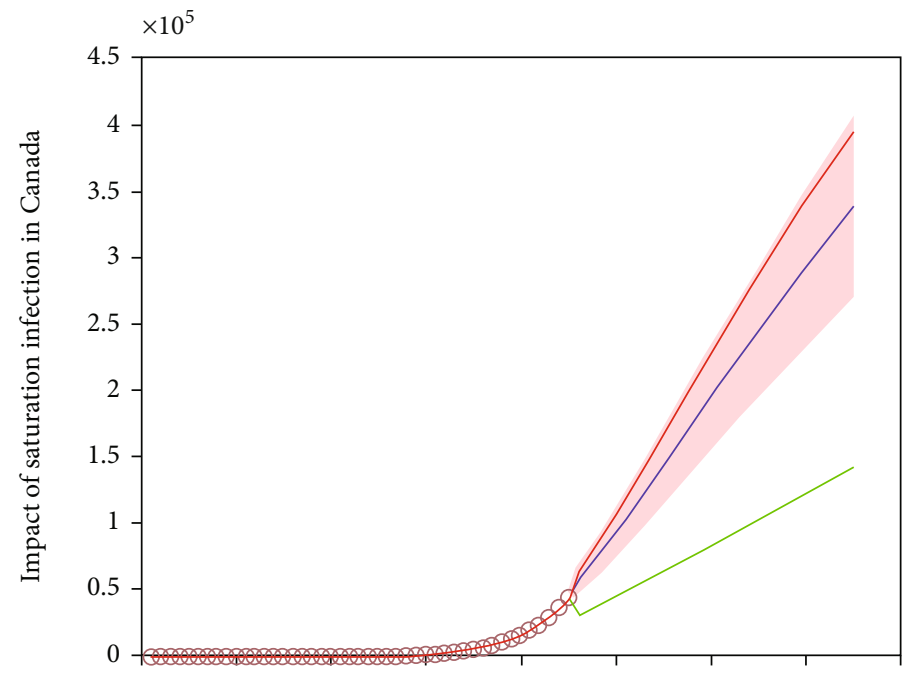

Feb 15 Feb 25 Mar 05 Mar 15 Mar 25 Apr 05 Apr 15 Apr 25 May 05

○ Data

$\alpha=0.00001$

$\alpha=0.00003$
Date (2020)

$\alpha=0.000008$

Confidence interval

(e)

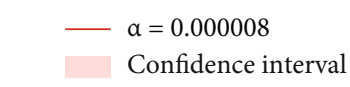

(f)

FIGURE 4: Impact of saturation infection $(\alpha)$ on the dynamics of COVID-19 cases in (a) Australia, (b) Italy, (c) Spain, (d) the USA, (e) the UK, and (f) Canada.

Moreover, Figure 4 shows the impact of saturation infection on the dynamics of COVID-19 cases in the six countries Australia, Italy, Spain, the USA, the UK, and Canada. We observed that saturation infection negatively correlates with
COVID-19 cases, which means increasing saturation infection will reduce the COVID-19 cases. Further, decreasing saturation infection will increase the COVID-19 cases in all six burden countries. 


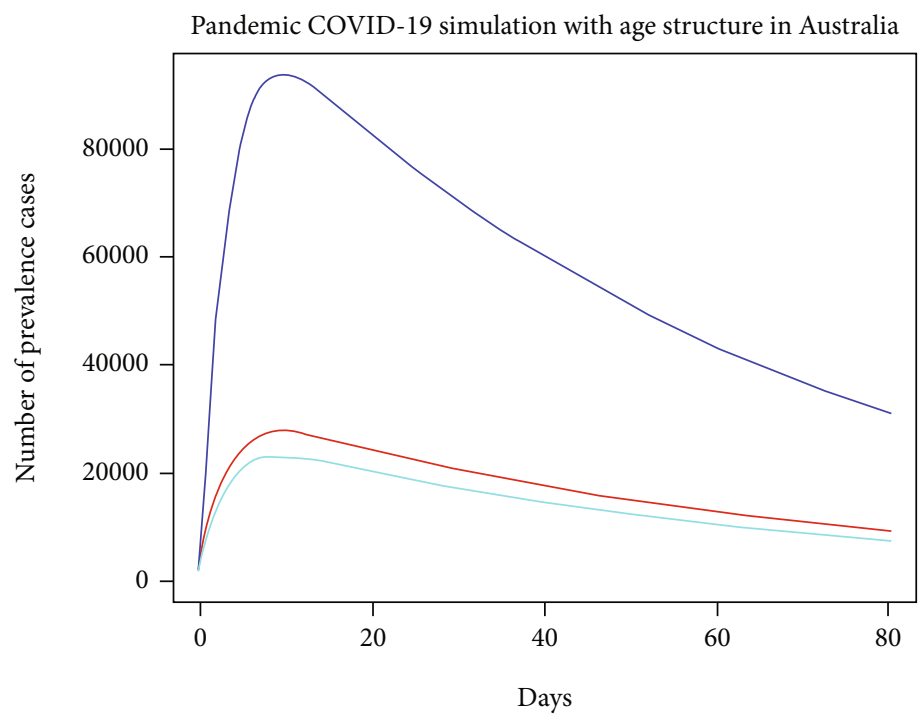

(a)

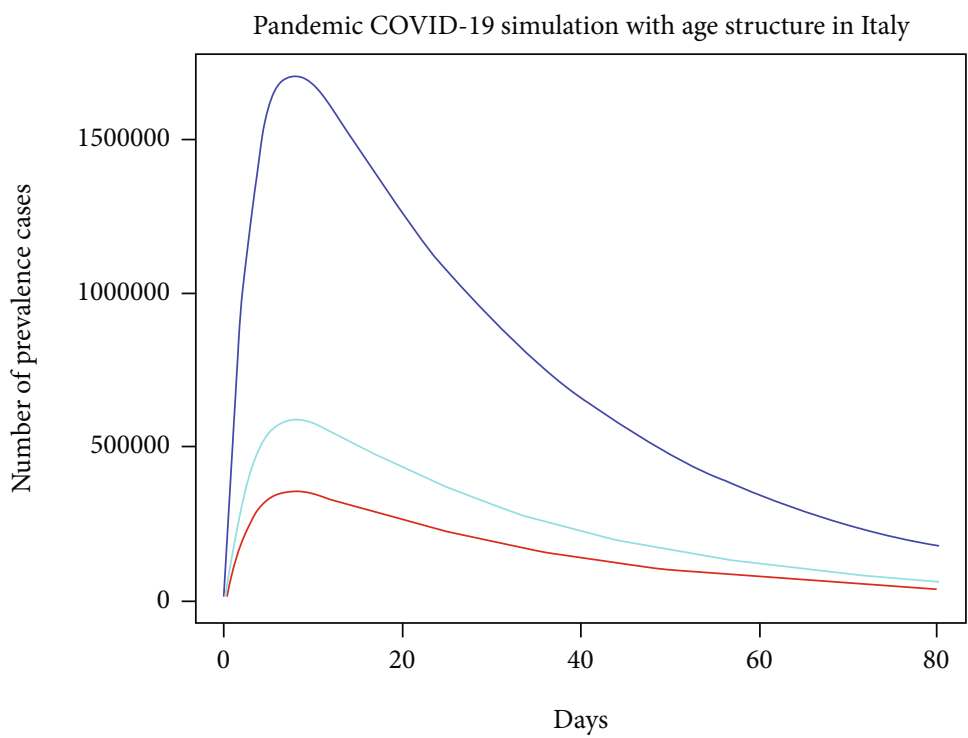

(b)

FIgURE 5: Continued. 


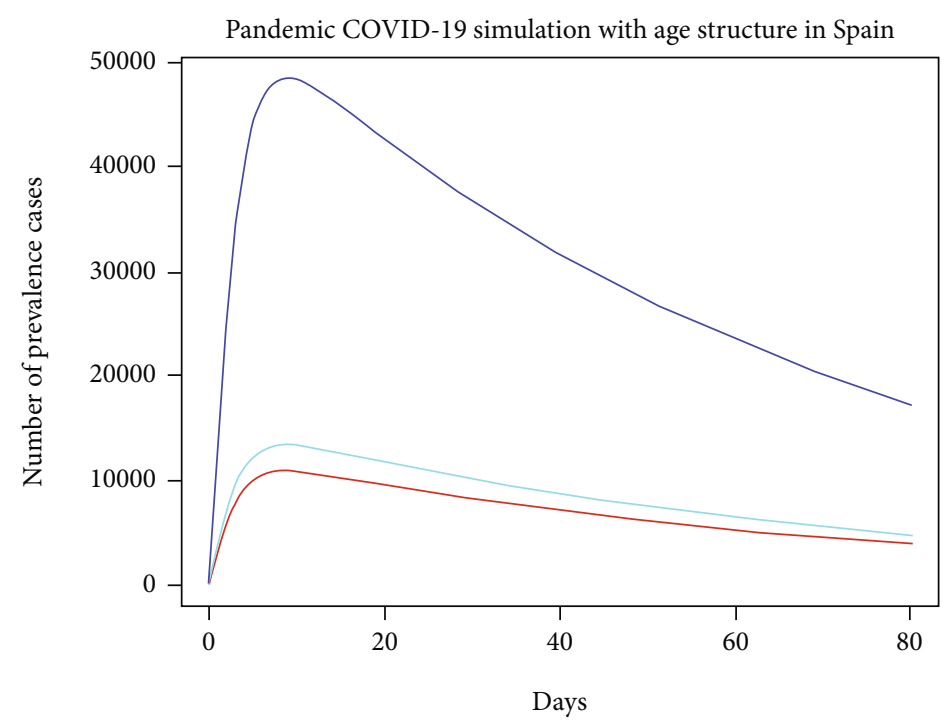

(c)

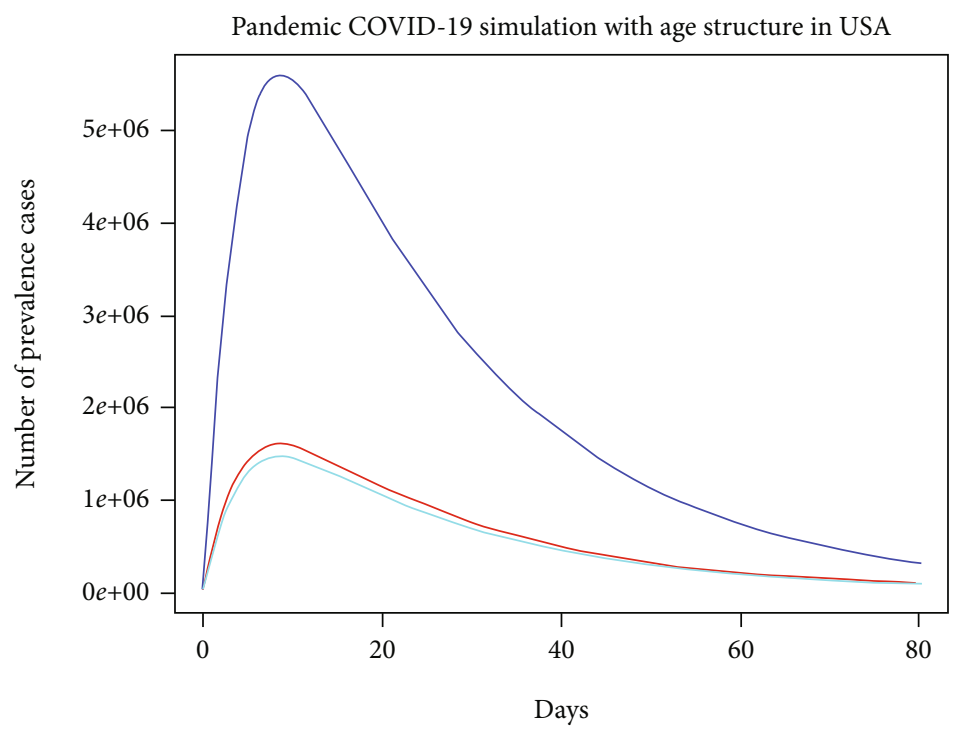

(d)

Figure 5: Continued. 


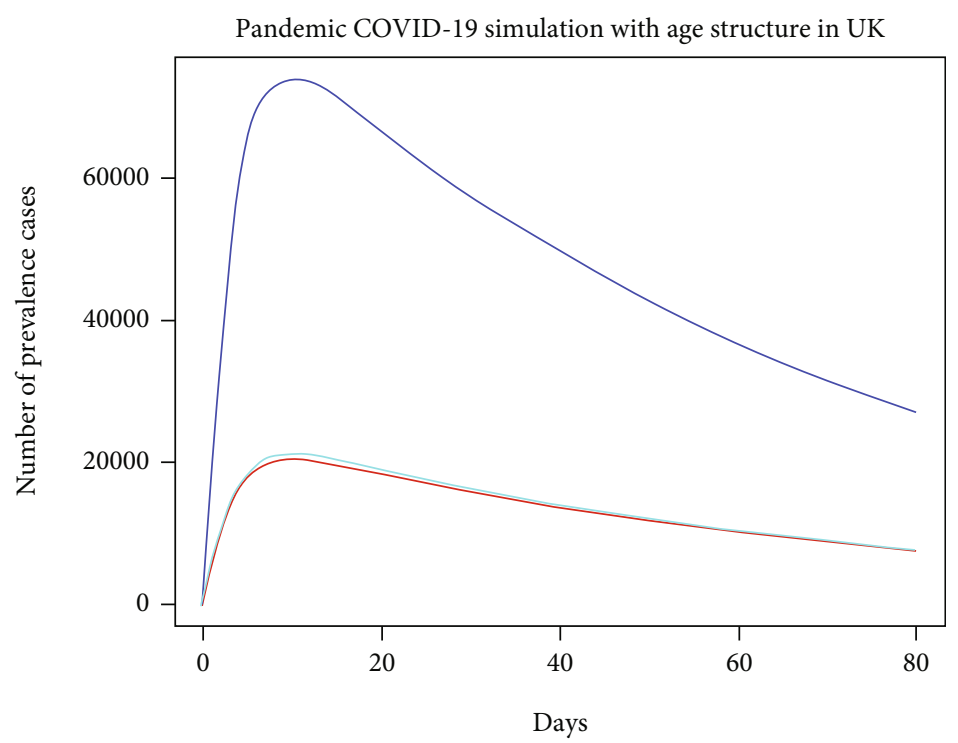

(e)

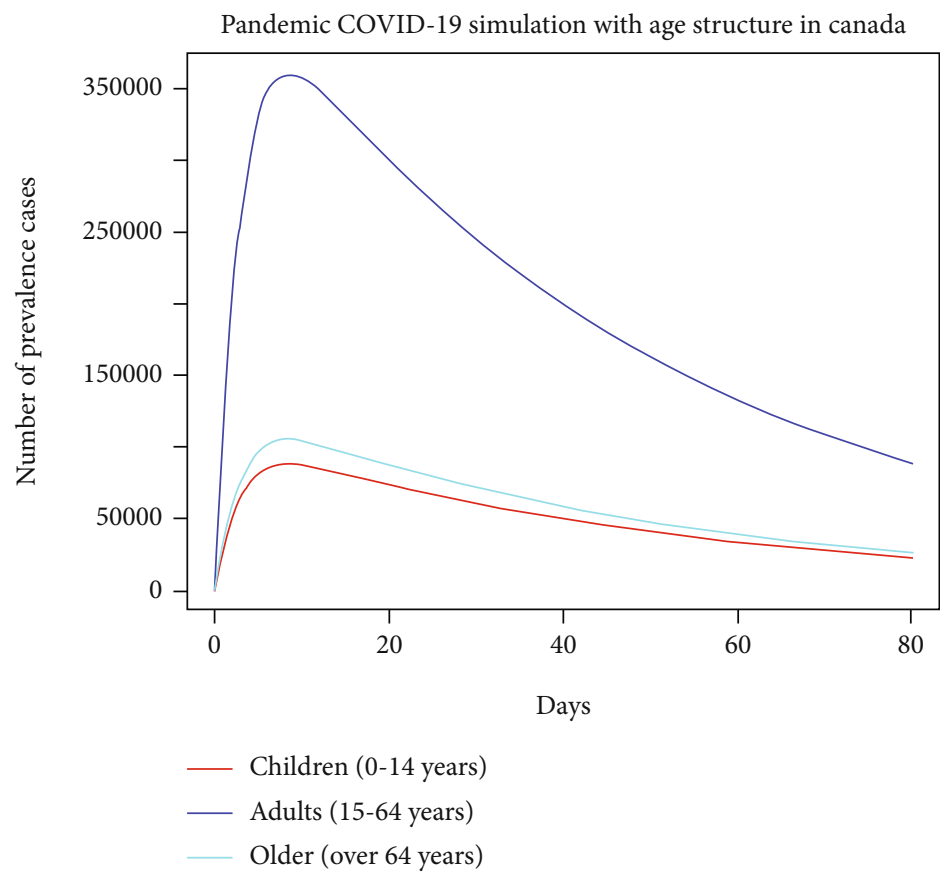

(f)

FIGURE 5: Shows the COVID-19 prevalence in each of the age groups as the epidemic progresses in six countries: (a) Australia, (b) Italy, (c) Spain, (d) the USA, (e) the UK, and (f) Canada.

\section{Impact of Age Structure on the Dynamics of COVID-19}

In this section, we explored the impact of three age classes, including children (0-14 years), adults (15-64 years), and older (over 64 years), on the transmission dynamics of COVID-19 cases over 80 days in the six high-burden countries. In Australia, Italy, Spain, the USA, the UK, and Canada, the percentages of children (0-14 years) among the total population are $18.72 \%, 13.45 \%, 15.02 \%$, $18.46 \%, 17.63 \%$, and $15.99 \%$, respectively. Further, the per- centages of adults (15-64 years) among the total population are $35.39 \%, 64.47 \%, 66.50 \%, 64.69 \%, 63.89 \%$, and $65.03 \%$, respectively. Finally, the percentages of older people (over 64 years) among the total population are $15.88 \%$, $22.08 \%, 18.49 \%, 16.85 \%, 18.48 \%$, and $18.98 \%$, respectively [22]. Results show that adults are more infected than children and the older population in all countries. However, in Italy, Spain, the UK, and Canada, older people are more infected than children. In contrast, children are more infected than older people in Australia and the USA (see Figure 5). 


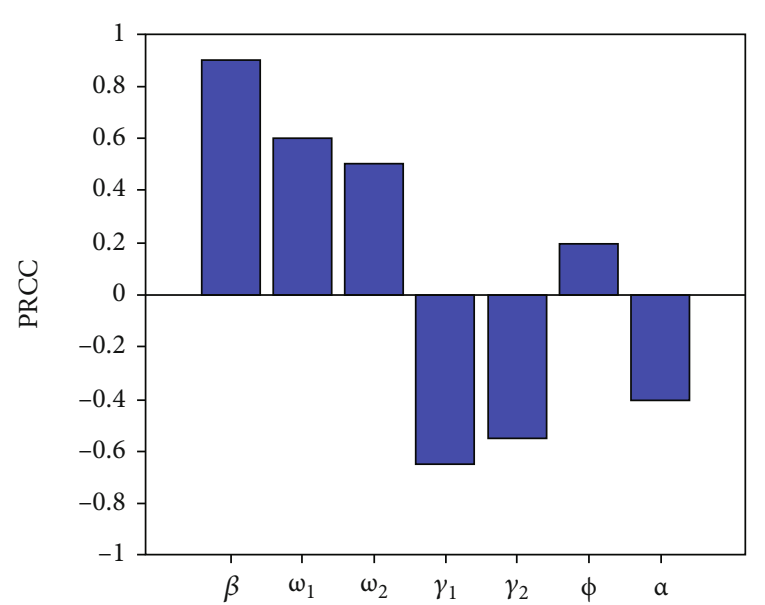

FIGURE 6: PRCC values depicting the sensitivities of the model output, i.e., the total number of infectious individuals $(M+C)$ for the estimated parameters $\beta, \omega_{1}, \omega_{2}, \gamma_{1}, \gamma_{2}, \phi$, and $\alpha$.

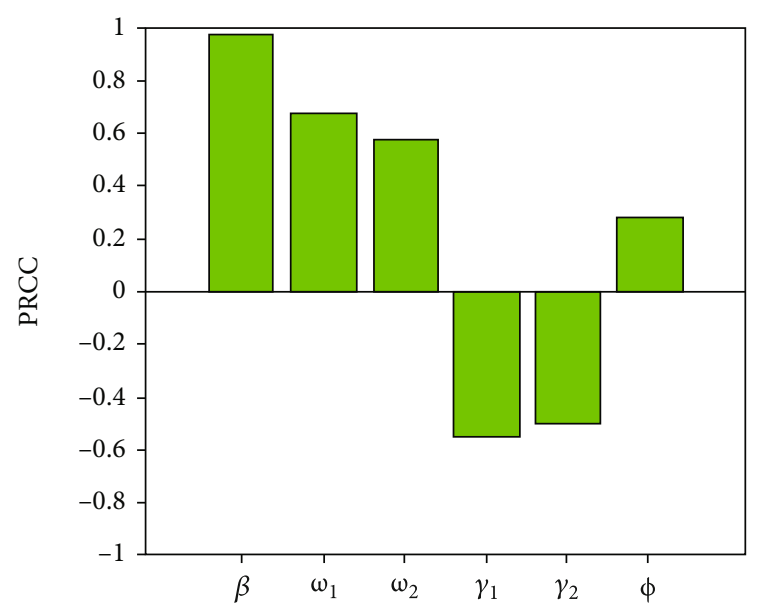

FIGURE 7: PRCC values depicting the sensitivities of the model output, i.e., the basic reproduction number $\left(R_{0}\right)$ for the estimated parameters $\beta, \omega_{1}, \omega_{2}, \gamma_{1}, \gamma_{2}$, and $\phi$.

\section{Sensitivity Analysis}

Sensitivity analysis is a handy method to investigate the parameters that most significantly influence on the model outputs [23, 24]. In this study, we performed the partial rank correlation coefficient (PRCC) estimation, a global sensitivity analysis technique proven to be the most reliable and efficient sampling-based method [24, 25]. The analysis conducted about 100,000 simulations by assigning a uniform distribution to each model parameter and using independent sampling. The positive (negative) correlation suggests that a positive (negative) variation in the parameter will increase (decrease) the model outcome [24]. Here, the model outputs we consider are the total number of infectious individuals $M+C$ (where, $M+C=\left(1+\left(\gamma_{2}\left(\phi+\gamma_{1}+\mu\right)+\phi \omega_{1}\right) / \omega_{1}\left(\gamma_{2}+\mu\right)\right)\left(\left(\beta \Lambda \omega_{1}^{2}\left(\omega_{1}\right.\right.\right.$ $\left.\left(\gamma_{2}+\mu\right)+\omega_{2}\left(\phi+\gamma_{1}+\mu\right)+\phi \omega_{1}\right)-\omega_{1} \mu\left(\gamma_{2}+\mu\right)\left(\omega_{1}+\omega_{2}+\mu\right)$ $\left.\left(\phi+\gamma_{1}+\mu\right)\right) /\left(\omega_{1}\left(\gamma_{2}+\mu\right)+\omega_{2}\left(\phi+\gamma_{1}+\mu\right)+\phi \omega_{1}\right)\left(\omega_{1}+\omega_{2}\right.$ $\left.\left.+\mu)\left(\phi+\gamma_{1}+\mu\right)\left(\alpha \omega_{1} \mu+\beta \omega_{1}\right)\right)\right)$ and the basic reproduction number $R_{0}$ (where, $R_{0}=(\Lambda / \mu) \beta \omega_{1} /\left(\omega_{1}+\omega_{2}+\mu\right)\left(\gamma_{1}+\phi+\mu\right)$ $+(\Lambda / \mu) \beta\left(\mu \omega_{2}+\omega_{1} \omega_{2}+\omega_{1} \phi+\omega_{2} \phi\right) /\left(\gamma_{2}+\mu\right)\left(\omega_{1}+\omega_{2}+\mu\right)($ $\left.\left.\gamma_{1}+\phi+\mu\right)\right)$. The PRCC results of $(M+C)$ and $R_{0}$ corresponding to the model parameters $\beta, \omega_{1}, \omega_{2}, \gamma_{1}, \gamma_{2}, \phi$, and $\alpha$ are displayed in Figures 6 and 7. Parameters $\beta, \omega_{1}, \omega_{2}$, and $\phi$ have positive PRCC values, implying that a positive change of these parameters will increase the total number of infectious individuals $(M+C)$ and the basic reproduction number. In contrast, parameters $\gamma_{1}, \gamma_{2}$, and $\alpha$ have negative PRCC values, which imply that increasing these parameters will decrease the total number of infectious individuals $(M+C)$ and $R_{0}$.

\section{Discussion and Concluding Remarks}

In this paper, we presented an age-structured SLMCR compartmental model with nonlinear incidence. We estimated the number of cases from COVID-19 infection and applied it to data from the COVID-19 epidemics in Australia, Italy, Spain, the USA, the UK, and Canada to predict the COVID-19 situation until $30^{\text {th }}$ April 2020 . After model calibration, we estimated the transmission rates of $4.6214 \times 10^{-8}, 1.2292 \times 10^{-8}, 3.3182$ $\times 10^{-7}, \quad 3.1828 \times 10^{-7}, \quad 1.1526 \times 10^{-7}$, and $2.0731 \times 10^{-7}$, respectively, in Australia, Italy, Spain, the USA, the UK, and Canada. The model estimates revealed a strong relationship between the transmission rate and the number of cases in COVID-19 of the selected countries, which is a consistent result with other studies related to COVID-19 [26-28].

Within the six selected countries, we found that Spain has the highest transmission rate than the other countries, which may increase the massive number of COVID-19 cases and make it one of the worst situations in Spain. The Spanish government may not have taken proper action at the initial stage to control transmission, including handwashing, social distancing, and good respiratory hygiene. For instance, in China, they took immediate action for transmission control, including lockdown in every city; that is how they were able to minimize the outbreak of COVID-19. Our finding is consistent with such typical observations because COVID-19 mainly spreads from person to person through droplet transmission. Droplets cannot go through the skin and can only lead to infection if they touch the mouth, nose, or eye. Therefore, it is essential to protect susceptible individuals from COVID-19 exposure from a public health perspective by effectively reducing the contact rate between susceptible and infectious individuals.

Age is a significant risk factor that can increase the severity of the outbreak of COVID-19. In our study, we found that adults are more infected than children and older people. The SARS-CoV-2 may be present for several days before there are any symptoms, and many adult people will have few or no symptoms at all. Surveillance data confirm that the SARS$\mathrm{CoV}-2$ virus is more in adults and had the highest rates of COVID-19 cases [29]. Modelling studies have found that children are less likely to acquire an infection and are much less likely to show symptoms [30], similar to our results in Italy, Spain, the UK, and Canada but dissimilar in Australia and the USA. This information will assist policymakers in strategy development. 
Estimation of transmission rates from different settings must be done with caution, though, as the pattern of a pandemic, the standard of care, and, as a result, the number of cases are time and setting dependent. For instance, very few cases have been reported so far in Bangladesh [31]. In this developing country, the health system is indigent and not comprehensive to cover every citizen, which leads to fewer reported cases. Therefore, data from other countries, in particular, the number of cases by date of COVID-19 onset, is necessary to better understand the variability in cases across different settings.

Our model determined that from the explicit expression for the total number of infectious individuals $(M+C)$ and the basic reproduction number $R_{0}$, it is clear that they depended on transmission rates $\beta$, progression rates $\omega_{1}$ and $\omega_{2}$, recovery rates $\gamma_{1}$ and $\gamma_{2}$, comorbidity rate $\phi$, and infection saturation rate $\alpha$. From the sensitivity analysis findings, it is also clear that the most critical parameter was the transmission rate, $\beta$, followed by the recovery rate, $\gamma_{1}$. Therefore, to control and eradicate COVID-19 infection, it is crucial to consider the following strategies: (i) the first and most important strategy is to minimize the contact rates $\beta$ with infected individuals by decreasing the values of $\beta$; (ii) the secondmost important strategy is to increase the recovery rate $\gamma_{1}$ of infective individuals through treatment. Therefore, we suggest that the most feasible and optimal strategy to eliminate COVID-19 in the six different countries, i.e., Australia, Italy, Spain, the USA, the UK, and Canada, is to reduce contact rates as well as increase the treatment rate. Finally, the application of the proposed model and its related outputs can be extended to many other countries dealing with such a critical outbreak of COVID-19 to control this global pandemic disease.

\section{Data Availability}

All data will be available upon request.

\section{Ethical Approval}

The study was based on aggregated COVID-19 surveillance data in Australia, Italy, Spain, the USA, the UK, and Canada taken from the worldometer. No confidential information was included because analyses were performed at the aggregate level. Therefore, no ethical approval is required.

\section{Conflicts of Interest}

The authors declare that there are no conflicts of interest.

\section{Authors' Contributions}

AR conceived, designed, and supervised the study. MAK contributed to the literature search and data collection. AR and MAK contributed to the data analysis, construction of table and figures, interpretation of results, and writing of the manuscript.

\section{Acknowledgments}

We acknowledge the editing support provided by the Data Science Research Unit (DSRU) at Charles Sturt University, Australia.

\section{References}

[1] Worldometer, Coronvirus cases and deaths, Dover, Delaware, USA, 2020.

[2] R. J. Maude, Y. Lubell, D. Socheat et al., "The role of mathematical modelling in guiding the science and economics of malaria elimination," International Health, vol. 2, no. 4, pp. 239-246, 2010.

[3] A. J. Kucharski, T. W. Russell, C. Diamond et al., "Early dynamics of transmission and control of COVID-19: a mathematical modelling study," 2020, https://www.medrxiv.org/ content/10.1101/2020.01.31.20019901v2/.

[4] A. Remuzzi and G. Remuzzi, "COVID-19 and Italy: what next?," The Lancet, vol. 395, no. 10231, pp. 1225-1228, 2020.

[5] L. J. White, J. A. Flegg, A. P. Phyo et al., "Defining the in vivo phenotype of artemisinin-resistant falciparum malaria: a modelling approach," PLoS medicine, vol. 12, no. 4, article e1001823, 2015.

[6] R. J. Maude, W. Pontavornpinyo, S. Saralamba et al., "The last man standing is the most resistant: eliminating artemisininresistant malaria in Cambodia," Malaria Journal, vol. 8, no. 1, p. 31, 2009.

[7] J. M. Trauer, R. Ragonnet, T. N. Doan, and E. S. McBryde, "Modular programming for tuberculosis control, the "AuTuMN" platform," BMC Infectious Diseases, vol. 17, no. 1, article 2648, p. 546, 2017.

[8] R. Ragonnet, J. M. Trauer, J. T. Denholm, B. J. Marais, and E. S. McBryde, "A user-friendly mathematical modelling web interface to assist local decision making in the fight against drugresistant tuberculosis," BMC Infectious Diseases, vol. 17, no. 1, p. 374, 2017.

[9] E. S. McBryde, M. T. Meehan, T. N. Doan et al., "The risk of global epidemic replacement with drug-resistant mycobacterium tuberculosis strains," International Journal of Infectious Diseases, vol. 56, pp. 14-20, 2017.

[10] C. I. Siettos and L. Russo, "Mathematical modeling of infectious disease dynamics," Virulence, vol. 4, no. 4, pp. 295-306, 2013.

[11] J. P. Vandenbroucke and N. Pearce, "Incidence rates in dynamic populations," International Journal of Epidemiology, vol. 41, no. 5, pp. 1472-1479, 2012.

[12] X.-Z. Li, W.-S. Li, and M. Ghosh, "Stability and bifurcation of an SIR epidemic model with nonlinear incidence and treatment," Applied Mathematics and Computation, vol. 210, no. 1, pp. 141-150, 2009.

[13] J. Yang, Y. Zheng, X. Gou et al., "Prevalence of comorbidities and its effects in patients infected with SARS-CoV-2: a systematic review and meta-analysis," International Journal of Infectious Diseases, vol. 94, pp. 91-95, 2020.

[14] V. MATLAB, 9.2. 0 (R2017a), The MathWorks Inc., Natick, MA, USA, 2017.

[15] Worldometer, Population of Australia, Dover, Delaware, USA, 2020. 
[16] Y. Yang, J. Li, Z. Ma, and L. Liu, "Global stability of two models with incomplete treatment for tuberculosis," Chaos Solitons Fractals, vol. 43, no. 1-12, pp. 79-85, 2010.

[17] Worldometer, Population of Italy, Dover, Delaware, USA, 2020.

[18] Worldometer, Population of Spain, Dover, Delaware, USA, 2020.

[19] Worldometer, Population of the United States, Dover, Delaware, USA, 2020.

[20] Worldometer, Population of United Kingdom, vol. 2020, Dover, Delaware, USA, 2020.

[21] Worldometer, Population of Canada, vol. 2020, Dover, Delaware, USA, 2020.

[22] I. Mundi, "Countries demographics profile," 2020, https:// www.indexmundi.com/factbook/countries.

[23] A. Rahman and A. Kuddus, "Cost-effective modeling of the transmission dynamics of malaria: a case study in Bangladesh," Communications in Statistics: Case Studies, Data Analysis and Applications, vol. 6, no. 2, pp. 270-286, 2020.

[24] S. Kim, A. A. de los Reyes V, and E. Jung, "Mathematical model and intervention strategies for mitigating tuberculosis in the Philippines," Journal of Theoretical Biology, vol. 443, pp. 100-112, 2018.

[25] J. B. Njagarah and F. Nyabadza, "Modelling optimal control of cholera in communities linked by migration," Computational and Mathematical Methods in Medicine, vol. 2015, Article ID 898264, 12 pages, 2015.

[26] S. L. Chang, N. Harding, C. Zachreson, O. M. Cliff, and M. Prokopenko, "Modelling transmission and control of the COVID-19 pandemic in Australia," 2020, https://arxiv.org/ abs/2003.10218/.

[27] V. Srivastava, S. Srivastava, G. Chaudhary, and F. Al-Turjman, "A systematic approach for COVID-19 predictions and parameter estimation," Personal and Ubiquitous Computing, no. article 1462, pp. 1-13, 2020.

[28] L. Wang, Y. Wang, D. Ye, and Q. Liu, "Review of the 2019 novel coronavirus (SARS-CoV-2) based on current evidence," International Journal of Antimicrobial Agents, vol. 55, no. 6, article 105948, 2020.

[29] T conversation, "COVID-19 cases are highest in young adults. We need to partner with them for the health of the whole community," 2020, https://theconversation.com/covid-19-casesare-highest-in-young-adults-we-need-to-partner-with-themfor-the-health-of-the-whole-community-144932.

[30] J. Mossong, N. Hens, M. Jit et al., "Social contacts and mixing patterns relevant to the spread of infectious diseases," PLoS Medicine, vol. 5, no. 3, article e74, 2008.

[31] Worldometer, Total coronavirus cases in Bangladesh, Dover, Delaware, USA, 2020. 